\title{
Melatonin inhibits the Migration of Colon Cancer RKO cells by Down-regulating Myosin Light Chain Kinase Expression through Cross-talk with p38 MAPK
}

\author{
Duo-Bing Zou ${ }^{1,2 \&}$, Xiao Wei ${ }^{1,2 \&}$, Ruo-Lei Hu ${ }^{1,2 *}$, Xiao-Ping Yang, ${ }^{1,2}$ Li Zuo ${ }^{1,2}$, \\ Su-Mei Zhang ${ }^{1,2}$, Hua-Qing Zhu ${ }^{1,2}$, Qing Zhou ${ }^{1,2}$, Shu-Yu Gui,3, Yuan Wang1,2*
}

\begin{abstract}
Background: Melatonin, which is mainly produced by the pineal gland, has a good inhibitory effect on cell growth of multiple cancer types. However, the underlying molecular mechanisms of anti-tumor activity for colon cancer have not been fully elucidated. In this study, we investigated the effects of melatonin on migration in human colon cancer RKO cells and the potential molecular mechanisms. Materials and Methods: The viability of RKO cells was investigated by MTT assay after treatment with melatonin, SB203580 ( $\mathbf{p 3 8}$ inhibitor) and phorbol 12-myristate 13-acetate (PMA, MAPK activator) alone or in combination for $48 \mathrm{~h}$. The effects of melatonin, and ML-7, a selective inhibitor of myosin light chain kinase (MLCK), and SB203580, and PMA on the migration of RKO cells were analyzed by in vitro scratch-wound assay. The relative mRNA levels of MLCK was assessed by real-time quantitative RT-PCR. Western blotting analysis was performed to examine the expression of MLCK, phosphorylation of myosin light chain (pMLC) and p38 (pp38). Results: The proliferation and migration of human colon cancer RKO cells were inhibited significantly after treatment with melatonin. The expression levels of MLCK and phosphorylation of MLC of RKO cells were reduced, and real-time quantitative RT-PCR showed that melatonin had significant effects on suppressing the expression of MLCK. Furthermore, the phosphorylation level of $\mathrm{p38}$, which showed the same trend, was also reduced when cells were treated by melatonin. In addition, ML-7 (25umol/l) could down-regulate the phosphorylation of p38. Conclusions: Melatonin could inhibit the proliferation and migration of RKO cells, and further experiments confirmed that 338 MAPK plays an important role in regulating melatonin-induced migration inhibition through down-regulating the expression and activity of MLCK.
\end{abstract}

Keywords: Melatonin - human colon cancer - MLCK - p38 MAPK - migration

Asian Pac J Cancer Prev, 16 (14), 5835-5842

\section{Introduction}

Human colon cancer is the most frequent cancer worldwide as well as in china, and which is a malignant tumor arising from the inner wall of the large intestine has become the third global leading cause of death from different cancers and its metastasis at distant sites is the major cause of death (André et al., 2004). It's particularly worth mentioning here that the majority of individuals who were diagnosed with this disease are already at an advanced stage (Jemal et al., 2011). Although colorectal tumors that are detected prior to invasion and metastasis can be eliminated with surgery, approximately half of resected colon cancer patients still experience tumor recurrence, and metastatic colon cancer after treatment with chemotherapy ultimately fails due to development of drug resistance (Vellinga et al., 2015). In the meantime, considering the reduction of drugs' toxicity and the prolong survival of patients with colon cancer, novel therapeutic strategies are urgently needed.

Melatonin (N-acetyl-5-methoxytryptamine), a kind of endogenous substances, is entrained by the light/dark cycle (Claustrat et al., 2005; Blask, 2009), and predominantly produced by the pineal gland (Reiter, 1991), and partly by other organs like the eyes, bone marrow, gastrointestinal tract, skin and lymphocytes (Claustrat et al., 2005; Srinivasan et al., 2011). For the past few years, melatonin has been attracting more and more attention as a potential new therapeutic method. Studies have shown that melatonin plays key roles in the biologic regulation of circadian rhythms, sleep, antioxidant protection, possibly aging, immune, tumor growth, reproduction and bone physiology (Verma et al., 2014; Akbulut et al., 2015; Plaimee et al., 2014a). Undoubtedly, More recent studies have implied that melatonin has the potentially of being used as an effect therapeutic agent for treating breast 
cancer, lung cancer, colorectal cancer, melanoma and various other types of cancer (Martin et al., 2006; Mao et al., 2010; Zha et al., 2012; Wang et al., 2013c; Zhou et al., 2014a). Moreover, melatonin could enhance the antitumor effect of some agents through modulating multiply signaling pathways in some cancer cells (Wang et al., 2013c; Yi et al., 2014). The inhibitory action of growth by melatonin in colon cancer has been studied extensively both in vivo and vitro in the last ten years (Wenzel et al., 2005; Garcia-Navarro et al., 2007; Tanaka et al., 2009; Winczyk et al., 2009). In contrast, only a minimal amount of work has been done with regard to the role of melatonin in human colon cancer invasion and metastasis. Therefore, elucidation of the detailed molecular mechanisms involved in melatonin-mediated inhibitory effect of migration in colon cancer deserves further investigation.

Myosin Light Chain Kinase (MLCK, dependent increases in myosin ATPase activity), a key $\mathrm{Ca}^{2+}$ / Calmodulin (CaM)-dmependent effector, is a myosin regulator in the lamella and contractile ring, and could regulate nonmuscle myosin II activity via phosphorylation of Ser19, Thr18 on myosin light chains (MLC) (Chew et al., 2002), and assemble in protrusive during cell migration (Kolega, 2003). It has been reported that the activation and increased expression of MLCK are crucial to trigger non-muscle cell and smooth muscle cell motility (EvenRam et al., 2007; Sun et al., 2011; Zhu et al., 2012). MLCK in cancer cell migration remains controversial. But, recently increasing studies have shown that increased expression levels of MLCK are crucial for migration activity in several types of human cancer (Kucharczak et al., 2001; Mills et al., 2011; Wang et al., 2013b; Fan et al., 2014). Furthermore, MLCK is not only required for myosin phosphorylation in a migrating cell, but also critical role of in cell migration involving regulating the cell membrane tension and protrusion necessary for migration (Chen et al., 2014). Mitogen-activated protein kinase (MAPK) signaling shows a cascade organization, in which activation of upstream kinases by receptors leads to sequential activation of a MAPK module (MAPKKK, MAPKK, MAPK), which play key roles in cell proliferation, differentiation, migration and cell survival (Joo et al., 2009; Mao et al., 2010; Liu et al., 2012). Recent researches and preliminary studies in our laboratory have shown that inhibiting tumor cells migration by down-regulating the expression of MLCK is associated with p38 signaling pathway (Wang et al., 2013a; 2013b), one of MAPKs pathway cascade. The MAPKs cascades include three classical signal pathways: extracellular signal-regulated protein kinases $1 / 2$ (ERK1/2 or MAPK p44/42), C-Jun-N-terminal kinases (JNK) and p38 MAPK. The p38 activation is closely related to cell proliferation, migration, and cell survival in various colorectal cancer cells (Cumaoglu et al., 2014; Del et al., 2014; Grossi et al., 2014). However, little is known whether melatonin is able to inhibit colon cancer RKO cell migration by blocking MLCK, in which the signaling pathways involved in mediation by melatonin have not been completely elucidated.

In the present study, we aimed to investigate the potential effects of melatonin on the migration of RKO cell and assess the expression of MLCK and the function of p38/MAPK signal transduction pathway. Eventually, our studies showed that the anti-proliferation and antimigration activity of melatonin in RKO cells were accompanied by inhibition of $\mathrm{p} 38$ MAPK pathways, and the inhibition of MLCK meditated by melatonin resulted in the depression of migration of colon cancer RKO cells through cross-talk between MLCK and down-regulated p38, in which both phosphorylated MLC serve as essential downstream effector. These results indicated that we have discovered a potent MLCK inhibitor that effectively blocks RKO cancer cell migration, and meanwhile a treatment might potentially become an effective way in colon cancer therapy.

\section{Materials and Methods}

Reagents and antibodies The human colon cancer cell lines RKO was obtained from American Type Culture Collection (ATCC, Manassas, VA, USA). Melatonin was provided by school of Pharmacy, Anhui Medical University (Anhui province, China). A specific p38 MAPK inhibitor SB203580 and PKC activator Phorbol 12-myristate 13-acetate (PMA) were purchased from Cayman Chemical (USA). 3- (4, 5-dimethylthiazol2-yl)-2, 5-diphenyl tetrazolium bromide (MTT) and Dimethyl Sulfoxide (DMSO) were obtained from sigma Chemical (USA). PMA, SB, and melatonin were dissolved in a small amount of DMSO before addition to the complete cell culture medium. Dulbecco's modified Eagle's medium (DMEM) was obtained from Gibco BRL life Technologies (USA). Fetal bovine serum was purchased from the Zhejiang Tianhang Biological Technology Co (China). Primary antibodies against MLCK, MLC, p-p38, p38, $\beta$-actin were purchased from Santa Cruz Biotechnology (USA). Antibody against $p$-MLC was purchased from Cell Signaling Technology. All secondary antibodies were purchased from Millipore (USA).

Cell culture Colorectal cancer RKO cells were cultured in DMEM high glucose culture media supplemented with $10 \%$ fetal bovine serum, $40 \mathrm{U} / \mathrm{ml}$ penicillin, and $100 \mathrm{U} / \mathrm{ml}$ streptomycin in an incubator with a humidified atmosphere of $5 \% \mathrm{CO}_{2}$ and $95 \%$ air at $37^{\circ} \mathrm{C}$.

Cell viability assay Cells were seeded in 96-well plates at a density of $5 \times 10^{\wedge} 3$ cells per well and were then continuously exposed to different combinations of melatonin, SB and PMA for $48 \mathrm{hr}$ at $37^{\circ} \mathrm{C}$. After that, MTT $(5 \mathrm{mg} / \mathrm{ml})$ dissolved in phosphate-buffered saline (PBS) was added to each well and incubated for 4 to $6 \mathrm{hr}$ at $37^{\circ} \mathrm{C}$. Then, the serum-free culture media containing MTT were discarded and DMSO was added to each well to dissolve the precipitate. The absorbance values were measured at $570 \mathrm{~nm}$ spectral wavelength using a Microplate reader after incubated and vibrated for $15 \mathrm{~min}$ at $37^{\circ} \mathrm{C}$.

Effect of melatonin on cell morphology Cell were seeded in 6-well plates, and then the next day treated with melatonin at $0.5 \mathrm{mM}, 1.5 \mathrm{mM}, 2.5 \mathrm{mM}$ for $48 \mathrm{hr}$. The cell morphology were photographed using a microscope (Leica DMI3000B).

Scratch-wound assays RKO cells were seeded in 12-well plates. After incubation when cell grow to $90 \%$ 
confluence, each well was manually scratched with a sterile $200 \mu$ l pipette tip, washed with $1 \times$ PBS three times and incubated at $37^{\circ} \mathrm{C}$ with the melatonin or combination of ML-7, SB and PMA. After treated for 12, 24 and $48 \mathrm{hr}$, the gap distance between two cell edges were measured using by Image-Pro Plus software.

Quantitative real time RT-PCR. Total RNA was extracted using the Trizol procedure (Invitrogen, USA). First-strand cDNA was synthesized from $4 \mu \mathrm{g}$ RNAusing the Reverse Transcription System (TAKARA). The resulting 4 $\mu \mathrm{g}$ cDNA was subjected to PCR amplification. Real-time PCRs were performed by using the SYBR Green QPCR master mixed with the following primer pairs: Human GAPDH (Fw: AGGTCGGAG TCAACGGATTTG; Rv:CCTGGAAGATGGTGATGGGAT). MLCK (Fw: GGACTTTCAGCCTTGTGATTC; Rv: CGCAAAACTTCCTTC TACTGTC). All primers were synthesized by the Sangon Biotech (Shanghai). Realtime PCR cycle conditions were as follows: an initial denaturation $95^{\circ} \mathrm{C}$ for $30 \mathrm{~s}$, followed by 40 cycles of a $5 \mathrm{~s}$ extension step at $95^{\circ} \mathrm{C}$, and annealing for $30 \mathrm{~s}$ at $60^{\circ} \mathrm{C}$.

Western Blot analysis After various treatments, the collected RKO cells was washed with PBS three times and lysed in RIPA lysis buffer (hepes $25 \mathrm{mM}, 1.5 \%$ Triton X-100, $1 \%$ sodium deoxycholate, $0.1 \% \mathrm{SDS}, \mathrm{NaCl}$ $0.5 \mathrm{M}$, EDTA $5 \mathrm{mM}$, NaF $50 \mathrm{mM}$, sodium vanadate 0.1 $\mathrm{mM}$, phenylmethylsulfonyl fluoride $1 \mathrm{mM}$, and leupeptin $0.1 \mathrm{~g} / \mathrm{l}, \mathrm{pH} 7.8$ ) on ice for $30 \mathrm{~min}$. The cell lysate was centrifuged at $14000 \mathrm{rpm}$ for $30 \mathrm{~min}$ at $4^{\circ} \mathrm{C}$ and the protein concentration in the supernatant was determined by BCA assay. Loading buffer (Tris- $\mathrm{HCl} 42 \mathrm{mM}, 10 \%$ glycerol, 2.3\% SDS, 5\% 2-mercaptoethanol and $0.025 \%$ bromophenol blue) was then added to the supernatant, which was subsequently boiled for $5 \mathrm{~min}$ and then electrophoresed on a $12.5 \%$ or $10 \%$ SDS-PAGE. Proteins were transferred to PVDF membrane and blocked the non-specific binding sites used PBST (PBS contained $0.05 \%$ Tween 20 ) with $5 \%$ nonfat dry milk for $2 \mathrm{hr}$, After that, the membrane was incubated with primary antibody (antibodies against pp38, p38, pMLC, MLC, MLCK) over night at $4^{\circ} \mathrm{C}$ and then with peroxidase-conjugated secondary antibodies in the second reaction at room temperature for $2 \mathrm{hr}$. Detection was performed with enhanced chemiluminescence sequentially reagent.

Statistical analysis Data were analyzed by a one-way analysis of variance (ANOVA) followed by The Dunnett t-test or LSD- test using SPSS16.0 software. All the experiments were carried out three times, and results are represented as means \pm standard deviation of the mean. Differences were determined to be significant with $\mathrm{P}<$ 0.05 .

\section{Results}

Effect of melatonin on viability in human colon cancer RKO cells

In the previously work, our data established the growth inhibitory and pro-apoptotic effects of melatonin on RKO cells. Melatonin slightly attenuated the number of RKO cell at low concentration (less than $1 \mathrm{mM}$ ) and significantly reduced the number at high concentration (more than
$2 \mathrm{mM}$ ) (data not shown). To ulteriorly explore the effect of melatonin on cell viability, RKO cells were treated with pharmacological inhibitor and activator to determine the role of p38 in the viability of RKO cells. The RKO cells were treated with SB203580, an inhibitor of p38, and PMA, an activator of PKC, for $48 \mathrm{hr}$. The results showed that SB203580 inhibited the viability of RKO cells and PMA had no obvious effect on the viability of RKO cells compared with the DMSO group, but interestly, PMA decreased the effects of melatonin and SB203580 on the viability of RKO cells $(p<0.05)$ (Figure 1A). These data suggest that melatonin may inhibit the proliferation of RKO cells in vitro through p38 MAPK signaling pathway.

Effect of melatonin on RKO cells morphology and spread

At the same time, we also detected the changes in cell morphology and spread of colon cancer RKO cells
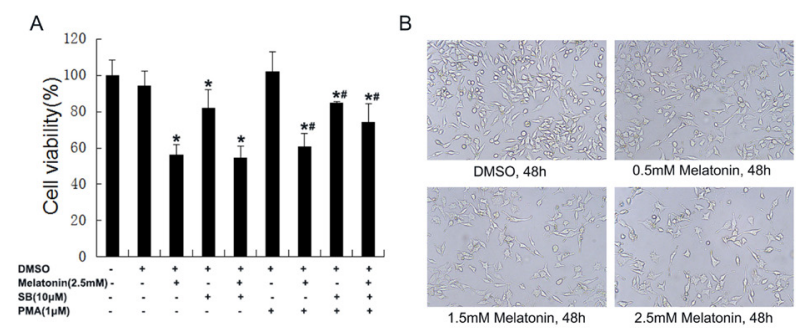

Figure 1. Effect of Melatonin, SB203580 and PMA on the Viability of RKO cells and morphology change. (A) The cells were treated with vehicle or melatonin $(2.5 \mathrm{mM})$, SB203580 $(10 \mu \mathrm{M})$ and PMA $(1 \mu \mathrm{M})$ alone, or in combination, for $48 \mathrm{hr}$, and cell viability was determined by a MTT assay. (B) The changes in cell morphology and spreading of RKO cells treated with different concentrations of melatonin for $48 \mathrm{hr}$ were observed, and cells were photographed using Leica DMI3000B. * $P<0.05$ compared with the cell group; ${ }^{\#} P<0.05$ compared with the PMA group

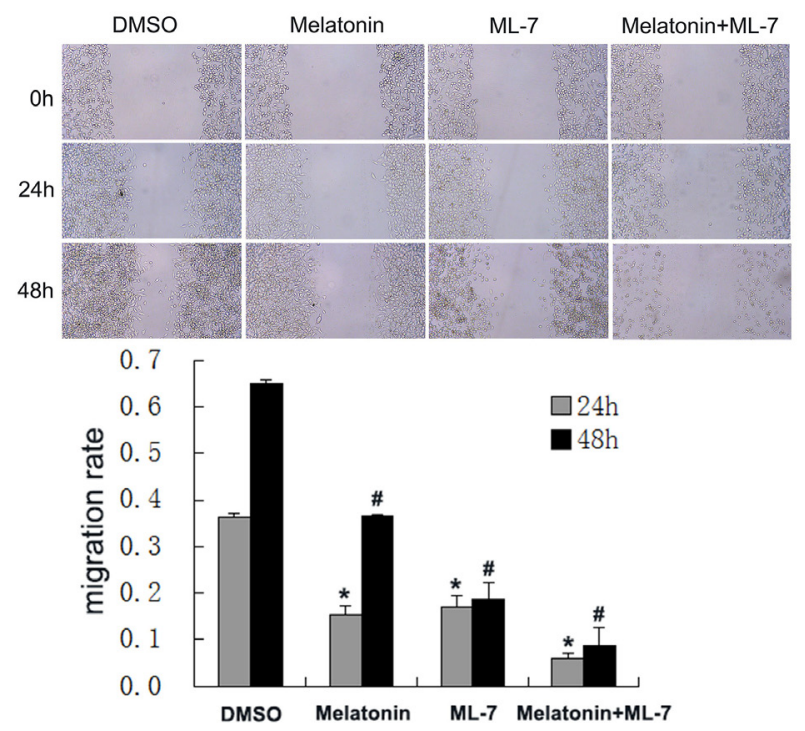
Figure 2. Effect of Melatonin and ML-7 on Migration of RKO Cells. After treating RKO cells with melatonin or ML-7 $(25 \mu \mathrm{M})$ alone, or in combination, for $48 \mathrm{hr}$, the treatment could block the migration of RKO cells. Calculating method: Relative migration ratio $=($ Start distance - End distance)/Start distance. ${ }^{*} P<0.05,{ }^{\#} P<0.05$ compared with the DMSO group. 


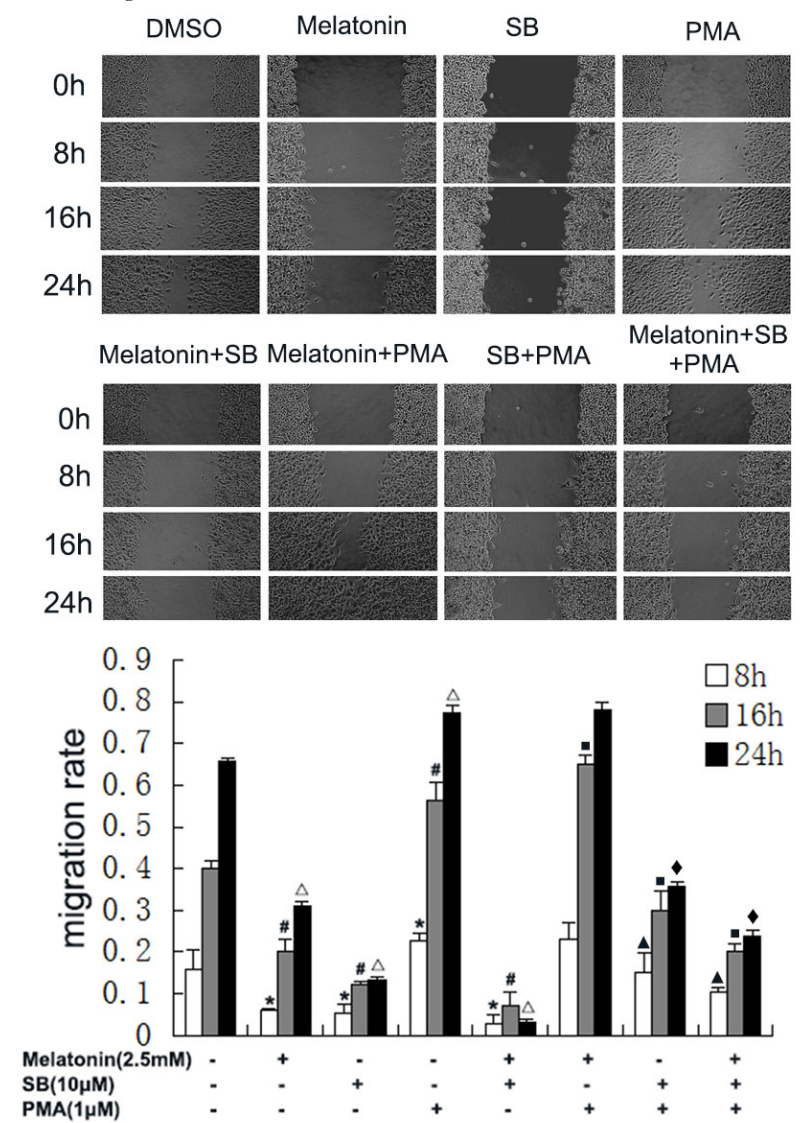

Figure 3. Effect of Melatonin, SB203580 and PMA on Migration of RKO Cells. The cells were treated with vehicle or melatonin $(2.5 \mathrm{mM})$, SB203580 $(10 \mu \mathrm{M})$ and PMA $(1 \mu \mathrm{M})$ alone, or in combination, for $24 \mathrm{hr}$.Melatonin and SB203580 significantly inhibited the migration of RKO cells, but PMA could partially prevented the decrease of melatonin and SB203580 on migration of RKO cells. ${ }^{*} P<0.05,{ }^{\sharp} P<0.05$, ${ }^{\Delta} P<0.05$ compared with DMSO group; ${ }^{\wedge} P<0.05,{ }^{-} P<0.05$, $\bullet P<0.05$ compared with PMA group

treated with melatonin. As shown in figure $1 \mathrm{~B}$, the control cells were easy to congregate closely with neatness and fusiform. After treated with melatonin for $48 \mathrm{hr}$, the treatment exhibited highly reduced cell-to-cell contact and was mostly individualized as compared with DMSO control groups. Meanwhile, the cells formed a cell layer and multiple antennae, and the more spread and filopodia were observed. What's more, the cells became sparser with wider intercellular spaces and irregular arrangement as compared with vehicle (Figure 1B). These changes after treated with melatonin might be associated with cell migration.

\section{Effect of melatonin on migration in RKO cells}

As our previous studies suggested that migration of RKO cells was inhibited by melatonin in a concentration dependent manner. To the further verify the correlation between cell migration and MLCK, we used ML-7 to treat RKO cells and observed the wound healing of RKO cells. ML-7 is a specific inhibitor of MLCK, which can specifically inhibit MLCK phosphorylating MLC. After being treated with ML-7 $(25 \mu \mathrm{M})$ for $24 \mathrm{hr}$ and $48 \mathrm{hr}$, the contrast migration rate of RKO cells was dramatically inhibited by ML-7 compared with the control group

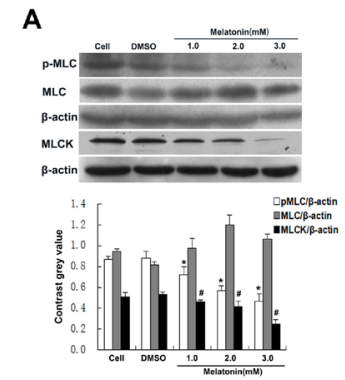

B
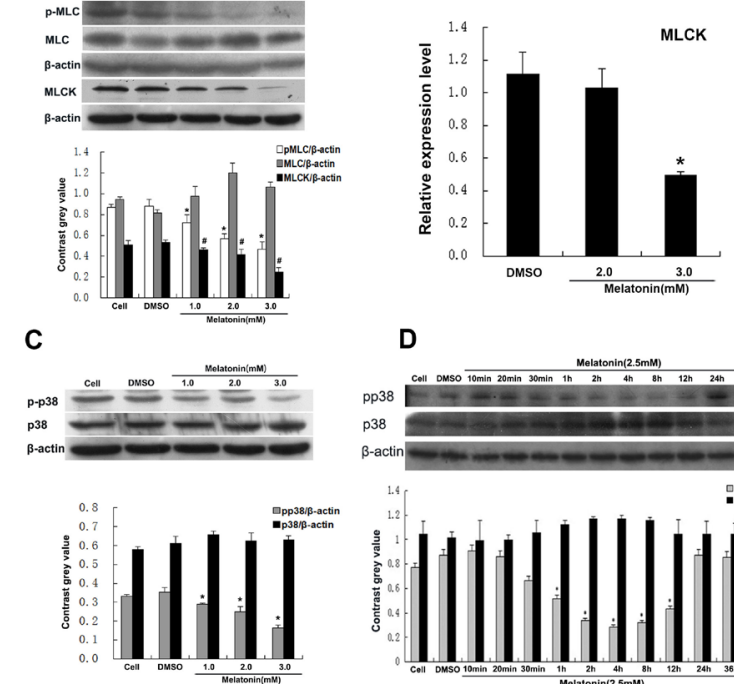

D

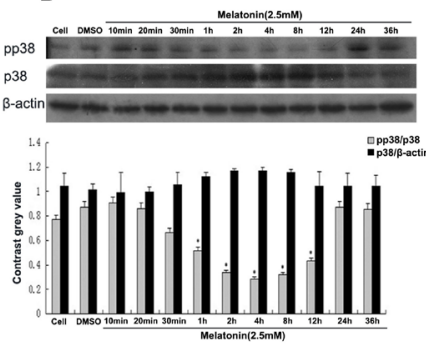

E
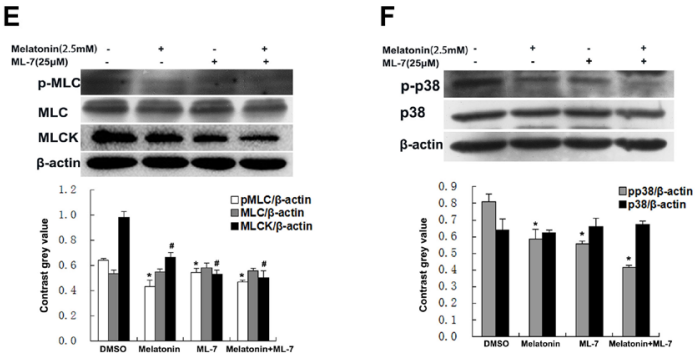

Figure 4. Effect of Melatonin, ML-7 on the Expression of MLCK and phosphorylation of MLC and p38. (A) After treating RKO cells with melatonin at different concentrations for $48 \mathrm{hr}$. The protein expression of MLCK and phosphorylation of MLC in RKO cells were decreased. (B) At the same time, the relative mRNA levels of MLCK was detected by real-time quantitative RT-PCR, which showed the same result. (C and D) Meanwhile, the phosphorylation of p38 was significantly decreased in a dose- and time-dependent manners. (E) ML-7 was used to treat RKO cells, and the expression of MLCK and phosphorylated of MLC were evidently decreased. (F) Furthermore, the phosphorylation of p38 was also decreased by ML-7. $* \mathrm{P}<0.05, \# \mathrm{P}<0.05$ compared with $\mathrm{DMSO}$ group

(Figure 2). We then used pharmacological inhibitor and activator to determine the role of p38 in the migration of RKO cells. Fig. 3 did not only show the similar outcomes of melatonin groups, but also unveiled that the effect of melatonin combining SB203580 on inhibiting cell migration was more remarkable than that of melatonin alone. The results showed that SB203580 significantly inhibited the migration of RKO cells and PMA promoted the migration of RKO cells but not very obviously compared with the control, however, PMA partially prevented the decrease of melatonin and SB203580 on migration of RKO cells (Figure 3).

Melatonin decreased expression of MLCK and phosphorylation of 38 and MLC in RKO cells

After the cells were treated with different concentrations of melatonin for $48 \mathrm{hr}$, the results revealed that melatonin sharply reduced the expression of MLCK and phosphorylation of MLC, especially at the concentration of $3 \mathrm{mM}$ (Figure 4A). Meanwhile, the relative mRNA 
A

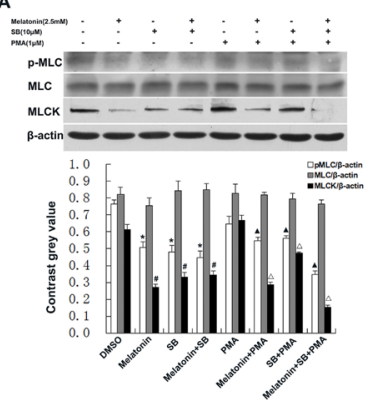

B

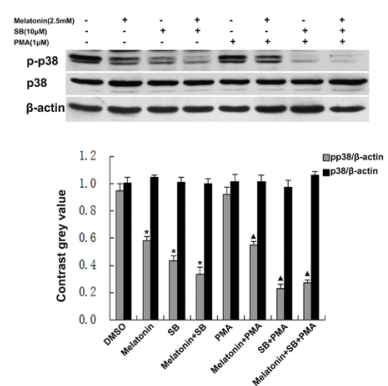

Figure 5. Effect of melatonin, SB203580 and PMA on the Expression of MLCK and Phosphorylation of MLC and p38. (A) The cells were treated with vehicle or melatonin, SB203580 and PMA alone, or in combination, for $48 \mathrm{hr}$. The expression of MLCK, the phosphorylated of MLC were down-regulated when cells were exposed to SB203580. Melatonin and SB203580 inhibited the expression of MLCK and phosphorylated of MLC in PMA-stimulated groups. (B) At the same time, the phosphorylation of $\mathrm{p} 38$ showed the same trend. ${ }^{*} P<0.05$, ${ }^{\sharp} P<0.05$ compared with DMSO group; $P<0.05,{ }^{\Delta} P<0.05$ compared with PMA group.

levels of MLCK was significantly decreased (Figure 4B). To evaluate the effect of melatonin on phosphorylation of p38, the level of phosphorylation of p38 in RKO cells treated by melatonin at various concentrations was measured by Western blot. Results showed that the level of phosphorylated p38 was decreased in a dose-dependent manner (Figure 4C). Furthermore, we also investigated the effect of melatonin $(2.5 \mathrm{mM})$ at different time points on the phosphorylation of p38 in RKO cells. Western blot showed that p38 phosphorylation, indicating p38 activation, was apparently decreased by melatonin-treated cells in a dose- and time-dependent manners (Figure $4 \mathrm{C}$ and D). As shown in Figure 4E, after treated RKO cells with ML-7 for $48 \mathrm{hr}$, the expression of MLCK and the phosphorylation of MLC were significantly decreased (Figure 4E). And we have extended the study and found that the phosphorylation of p38 was decreased when the cells were treated with ML-7 (Figure 4F).

Melatonin down-regulates the expression of MLCK in RKO cells, which is partly through the p38/MAPK signaling pathway

After that, we conjectured that whether the expression and activity of MLCK is associated with p38/MAPK. To determine whether signaling pathway related to $\mathrm{p} 38$ was involved in melatonin-induced migration suppression, the effect of PKC activator (PMA) and p38 inhibitor (SB203580) on expression of MLCK was examined. Western blot analysis showed that melatonin inhibited the expression of MLCK and the phosphorylation of MLC as well as SB203580. Combination of melatonin and SB further reduced MLCK expression. Treatment with PMA partly prevented the decrease of the MLCK as compared to the melatonin-treated group (Figure $5 \mathrm{~A})$. At the same time, figure 5B showed that melatonin induced a significant reduction of $\mathrm{p} 38$ phosphorylation as compared to the control group. SB itself greatly reduced

the p38 phosphorylation. Combination of melatonin and SB further reduced the phosphorylation of $\mathrm{p} 38$ to a very low level. What's more, the cells were coincubated with melatonin and PMA. We found that activation of PKC by PMA partially prevented the decrease of the p38 phosphorylation induced by melatonin (Figure 5B). Taken together, our findings indicated that melatonin induced the down-regulation of p38 MAPK signaling pathway in a dose- and time-dependent manner, suggesting that $\mathrm{p} 38$ MAPK may be specifically suppressed the migration of RKO cells by decreasing the expression and activity of MLCK.

\section{Discussion}

Cancer biological process is tightly associated with cell migration and invasion (Arafat et al., 2013; Pitchakarn et al., 2013). And the malignant process of metastasis has several components, including the cell proliferation, ability to invade through the acquisition of cell motility, degradation of basement membranes and extracellular matrices, and survival signaling. But, for current anticancer drugs mainly target tumour growth, finding a way to subdue cancer cell invasion, particularly in an adjuvant setting, molecularly-targeted inhibitors that blocked key invasion drivers would be expected to provide clinical benefit to a significant range of cancer patients with solid tumours at various stages. Melatonin is well-known indoles hormone mainly product secreted by the pineal gland in cyclical periods and a widely used antioxidant drug (Katzer et al., 2015; Shin et al., 2014; Borges et al., 2015). In addition, melatonin has been attracting more and more attention by exerting anti-proliferative, proapoptotic, and anti-angiogenic properties in multiple types of cancer cells (Lee et al., 2014; Plaimee et al., 2014b; Yi et al., 2014; Yun et al., 2014). And recently, an increasing number of studies have showed that melatonin could repress migration in various cancer cells (Wang et al., 2012d; Ordoñez et al., 2014; Zhou et al., 2014a). However, the effects and underlying molecular mechanisms of melatonin on colorectal cancer RKO cells migration inhibition are still not fully understood. Mechanically, we presented that melatonin might inhibit the proliferation and migration of RKO cells via down-regulating the phosphorylation of $\mathrm{p} 38$, and moreover, whether melatonin affected the migration of human colon cancer RKO cell via MLCK and through p38 MAPK pathway. To test this hypothesis, we first analyzed the effect of melatonin on tumor cell proliferation and migration in colon cancer RKO cells. Next, we analyzed the combined effects of SB203580, PMA, ML-7, and melatonin on some key proteins involved in cell proliferation and migration signaling pathways to uncover the molecular mechanisms of the natural products in colon cancer cells.

The morphology of tissue cells, acquire the ability for invasion mostly under special conditions, especially in the course of neoplastic evolution.The acquisition of cell locomotion, including migration and invasion, is closely associated with dynamic morphology of this cell and, especially with the dynamics of its cytoskeletal 
and adhesive structures (Vasiliev et al., 2004; VicenteManzanares et al., 2007). And driving force for movement is mainly established by a dynamic structural balance between two kinds of cytoskeletal Arrangements: microfilaments and focal adhesions, whereas the structural balance of cancer cells is lost. In this paper, we have briefly discuss the influence of morphological changes in migration.After RKO cells were treated with melatonin for $48 \mathrm{hr}$, we found that the melatonin inhibited ruffle formation and increased cell anchorage possibly by augmenting the number and thickness of stress fibers, as well as focal adhesions (Figure 1B). The results supported that the cytoskeleton may be a therapeutic target for melatonin to block invasiveness and metastasis formation in cancer cells. In particular, some studies also confirmed the same results (Vasiliev et al., 2004; Ortiz-Lopez et al., 2009). Increasing evidences indicated that myosin light chain kinase (MLCK) and mitogenactivated protein kinase signaling, in addition to their roles in the control of proliferation and cell death, are also implicated in the regulation of cell motility (Viala et al., 2004; Zhou et al., 2008b). It is known to us that MLCK is subsequently activated by the increase of the intracellular $\mathrm{Ca} 2+$. Recent studies have also suggested that MLCK inhibitors could be useful as anti-cancer agents. Because MLC phosphorylation, which is activated by MLCK, is essential to trigger actin-myosin interaction (Lai et al., 2003). This interaction in smooth muscle and non-muscle cells is regulated by MLC phosphorylation. Consequently, changes in MLC phosphorylation should affect the ability of cells to divide, but also result in cell migration (Wilson et al., 1991; Klemke et al., 1997). In our study, we investigated the signal pathways involved in the inhibition of proliferation and migration of colon cancer cells mediated by melatonin using RKO cell line as a model.we found that the treatment with melatonin could potently inhibit the proliferation and migration of RKO cells by cell viability assay and vitro scratch- wound assay (Figure 1A and 2), and western blotting assay indicated that the expression of MLCK and phosphorylation of MLC were markedly decreased compared with control group (Figure 4). Studies have been reported that treatment with ML-7, a specific inhibitor of MLCK, was able to inhibit the proliferation and migration of tumor cells through blocking MLCK (Kaneko et al., 2002). Moreover, ML-7 was used to treat RKO cells and the similar result that ML-7 could more notably suppress RKO cells migration revealed that melatonin suppressed RKO cells migration by down-regulating the expression of MLCK and phosphorylation of MLC. Furthermore, recently, several studies have shown that MLCK can also retard the proliferation of tumor cells (Gu et al., 2006; Zhou et al., 2008 b). Our studies also discovered that the proliferation of RKO cells was inhibited, the phosphorylation of p38 was decreased by ML-7, which showed the same trend as MLCK, suggesting that MLCK may also contribute to the proliferation of colon cancer RKO cells through cross-talk with inhibited p38/MAPK. p38, one of MAPKs signal cascade, is associated with the proliferation and migration properties of tumor cells as a prominent signal pathway (Wang et al., 2013a; Arechederra et al., 2015;
Yan et al., 2015). But, the detailed mechanisms of p38/ MAPK together with MLCK in colon cancer RKO cell migration have not been clarified. To determine the mechanism, we used PMA and SB203580 to respectively activate and inhibit MAPK/p38 signaling pathway in RKO cells. The results we did as shown in Figure 3, the migratory ability of colon cancer RKO cells was inhibited by melatonin and SB, but the decrease was prevented by PMA (Figure 3). Meanwhile, The expression of MLCK and the phosphorylated of MLC were down-regulated when cells were exposed to SB203580. Melatonin and SB203580 inhibited the expression of MLCK and phosphorylated of p38, MLC in PMA-stimulated groups (Figure 5). Therefore, in accordance with our data, the anti-migration effect of melatonin is associated with its inhibition of p38 MAPK pathway and regulation of the expression of MLCK.

Taken altogether, our present results strongly implied that p38 MAPK played an important role in inhibiting the proliferation and migration of RKO cells by melatonin. Down-regulation of pp38 levels by melatonin may contributed to RKO cell migration through decreasing the expression of MLCK and the activation of MLC, and inhibiting myosin light chain kinase possibly retards the growth of colon cancer RKO cells.Inhibition of p38 MAPK may be an important therapeutic target aimed at preventing the migration of colon cancer cell, suggesting that a treatment might potentially become an effective way in colon cancer therapy. Further researches are still needed to uncover the comprehensive molecular mechanisms of the natural products in tumor cells migration, because of the complex process which involves many factors and multiple steps.

\section{References}

Akbulut KG, Aktas SH and Akbulut H (2015). The role of melatonin, sirtuin 2 and FoXO1 transcription factor in the aging process of colon in male rats. Biogerontol, 16, 99-108.

André T, Boni C, Mounedji-Boudiaf L, et al (2004). Oxaliplatin, fluorouracil, and leucovorin as adjuvant treatment for colon cancer. $N$ Engl J Med, 350, 2343-51.

Arafat K, Iratni R, Takahashi T, et al (2013). Inhibitory effects of salinomycin on cell survival, colony growth, migration, and invasion of human non-small cell lung cancer A549 and LNM35: Involvement of NAG-1. PLoS One, 8, 66931.

Arechederra M, Priego N, Vázquez-Carballo A, et al (2015). p38 MAPK down-regulates fibulin 3 expression through methylation of gene regulatory sequences: Role in migration and invasion. J Biol Chem, 290, 4383-97.

Blask DE (2009). Melatonin, sleep disturbance and cancer risk. Sleep Medicine Rev, 13, 257-64.

Borges Lda S, Dermargos A, Junior EP, et al (2015). Melatonin decreases muscular oxidative stress and inflammation induced by strenuous exercise and stimulates growth factor synthesis. J Pineal Res, 58, 166-72.

Chen C, Tao T, Wen C, et al (2014). Myosin Light Chain Kinase (MLCK) Regulates Cell Migration in a Myosin Regulatory Light Chain Phosphorylation-Independent Mechanism. J Biol Chem, 289, 28478-88.

Chew TL, Wolf WA, Gallagher PJ, et al (2002). A fluorescent resonant energy transfer-based biosensor reveals transient and regional myosin light chain kinase activation in lamella and cleavage furrows. $J$ Cell Biol, 156, 543-53. 
Claustrat B, Brun J, and Chazot G (2005). The basic physiology and pathophysiology of melatonin. Sleep Med Rev, 9, 11-24.

Cumaoglu A, Dayan S, Agkaya AO, et al (2014). Synthesis and pro-apoptotic effects of new sulfonamide derivatives via activating p38/ERK phosphorylation in cancer cells. $J$ Enzyme Inhib Med Chem, 8, 1-7.

Del Reino P, Alsina-Beauchamp D, Escos A, et al (2014). Prooncogenic role of alternative $\mathrm{p} 38$ mitogen-activated protein kinases $\mathrm{p} 38 \gamma$ and $\mathrm{p} 38 \delta$, linking inflammation and cancer in colitis-associated colon cancer. Cancer Res, 74, 6150-60.

Even-Ram S, Doyle AD, Conti MA, et al (2007). Myosin IIA regulates cell motility and actomyosin-microtubule crosstalk. Nat Cell Biol, 9, 299-309.

Fan TT, Cheng Y, Wang YF, et al (2014). A Novel All-trans Retinoid Acid Derivative N- (3-trifluoromethyl-phenyl)Retinamide Inhibits Lung Adenocarcinoma A549 Cell Migration through Down-regulating Expression of Myosin Light Chain Kinase. Asian Pac J Cancer Prev, 15, 7687-92.

Garcia-Navarro A, González-Puga C, Escames G, et al (2007). Cellular mechanisms involved in the melatonin inhibition of HT-29 human colon cancer cell proliferation in culture. J Pineal Res, 43, 195-205.

Grossi V, Peserico A, Tezil T, et al (2014). p38 $\alpha$ MAPK pathway: a key factor in colorectal cancer therapy and chemoresistance. World J Gastroenterol, 20, 9744-58.

Gu LZ, Hu WY, Antic N, et al (2011). Inhibiting myosin light chain kinase retards the growth of mammary and prostate cancer cells. Eur J Cancer, 42, 948-57.

Jemal A, Bray F, Center MM, et al (2011). Global cancer statistics. CA Cancer J Clin, 61, 6990.

Joo SS, and Yoo YM (2009). Melatonin induces apoptotic death in LNCaP cells via p38 and JNK pathways: therapeutic implications for prostate cancer. J Pineal Res, 47, 8-14.

Kaneko K, Satoh K, Masamune A, et al (2002). Myosin light chain kinase inhibitors can block invasion and adhesion of human pancreatic cancer cell lines. Pancreas, 24, 34-41.

Katzer D, Mueller A, Welzing L, et al (2015). Antioxidative status and oxidative stress in the fetal circulation at birth: the effects of time of delivery and presence of labor. Early Hum Dev, 91, 119-24.

Klemke RL, Cai S, Giannini AL, et al (1997). Regulation of cell motility by mitogen-activated protein kinase. J Cell Biol, 137, 481-92.

Kolega J (2003). Asymmetric distribution of myosin IIB in migrating endothelial cells is regulated by a rho-dependent kinase and contributes to tail retraction. Mol Biol Cell, 14, 4745-57.

Kucharczak J, Pannequin J, Camby I, et al (2001). Gastrin induces over-expression of genes involved in human U373 glioblastoma cell migration. Oncogene, 20, 7021-28.

Lai JM, Hsieh CL, and Chang ZF (2003). Caspase activation during phorbol ester-induced apoptosis requires ROCKdependent myosin-mediated contraction. J Cell Sci, 116, 3491-501.

Lee YJ, Lee JH, Moon JH, and Park SY (2014). Overcoming hypoxic-resistance of tumor cells to TRAIL-induced apoptosis through melatonin. Int J Mol Sci, 15, 11941-56.

Liu L, Zhu Y, Xu Y and Reiter RJ (2012). Prevention of ERK activation involves melatonin-induced $\mathrm{G} 1$ and $\mathrm{G} 2 / \mathrm{M}$ phase arrest in the human osteoblastic cell line hFOB 1.19. J Pineal Res, 53, 60-6.

Mao L, Yuan L, Slakey LM, et al (2010). Inhibition of breast cancer cell invasion by melatonin is mediated through regulation of the p38 mitogen-activated protein kinase signaling pathway. Breast Cancer Res, 12, 107.

Martin V, Herrera F, Carrera-Gonzalez P, et al (2006). Intracellular Signaling Pathways Involved in the Cell
Growth Inhibition of Glioma Cells by Melatonin. Cancer Res, 66, 1081-8.

Mills AM, Beck AH, Montgomery KD, et al (2011). Expression of subtype-specific group 1 leiomyosarcoma markers in a wide variety of sarcomas by gene expression analysis and immunohistochemistry. Am J Surg Pathol, 35, 583-9.

Ordoñez R, Carbajo-Pescador S, Prieto-Dominguez N, et al (2014). Inhibition of matrix metalloproteinase-9 and nuclear factor kappa B contribute to melatonin prevention of motility and invasiveness in HepG2 liver cancer cells. J Pineal Res, 56, 20-30.

Ortiz-Lopez L, Morales-Mulia S, Ramirez-Rodriguez G and Benitez-King G (2009). ROCK-regulated cytoskeletal dynamics participate in the inhibitory effect of melatonin on cancer cell migration. J Pineal Res, 46, 15-21.

Pitchakarn P, Chewonarin T, Ogawa K, et al (2013). Ellagic Acid inhibits migration and invasion by prostate cancer cell lines. Asian Pac J Cancer Prev, 14, 2859-63.

Plaimee P, Khamphio M, Weerapreeyakul N, et al (2014a). Immunomodulatory effect of melatonin in SK-LU-1 human lung adenocarcinoma cells co-cultured with peripheral blood mononuclear cells. Cell Prolif, 47, 406-15.

Plaimee P, Weerapreeyakul N, Thumanu K, et al (2014b). Melatonin induces apoptosis through biomolecular changes, in SK-LU-1 human lung adenocarcinoma cells. Cell Prolif, 47, 564-77.

Reiter RJ (1991). Pincal melatonin: cell biology of its synthesis and of its physiological interactions. Endocr Rev, 12, 151-80.

Shin EJ, Chung YH, Le HL, et al (2014). Melatonin attenuates memory impairment induced by klotho gene deficiency via interactive signaling between MT2 receptor, ERK and Nrf2related antioxidant potential. Int J Neuropsychopharmacol, $18,105$.

Srinivasan V, Pandi-Perumal SR, Brzezinski A, et al (2011). Melatonin, Immune Function and Cancer. Recent Pat Endocr Metab Immune Drug Discov, 5, 109-23.

Sun C, Wu MH, and Yuan SY (2011). Nonmuscle myosin light-chain kinase deficiency attenuates atherosclerosis in apolipoprotein E-deficient mice via reduced endothelial barrier dysfunction and monocyte migration. Circulation, 124, 48-57.

Tanaka T, Yasui Y, Tanaka M, et al (2009). Melatonin suppresses AOM/DS -induced large bowel oncogenesis in rats. Chem Biol Interact, 177, 128-36.

Vasiliev JM (2004). Cytoskeletal mechanisms responsible for invasive migration of neoplastic cells. Int J Dev Biol, 48, 425-39.

Vellinga TT, Borovski T, de Boer VC, et al (2015). SIRT1/ PGC1 $\alpha$ dependent increase in oxidative phosphorylation supports chemotherapy resistance of colon cancer. Clin Cancer Res, 21, 2870-9

Verma D, Hashim OH, Jayapalan JJ, and Subramanian P (2014). Effect of melatonin on antioxidant status and circadian activity rhythm during hepatocarcinogenesis in mice. $J$ Cancer Res Ther, 10, 1040-4.

Viala E, and Pouysségur J et al (2004). Regulation of tumor cell motility by ERK mitogen-activated protein kinases. Ann $N$ $Y$ Acad Sci, 1030, 208-18.

Vicente-Manzanares M, Zareno J, Whitmore L, et al (2007). Regulation of protrusion, adhesion dynamics, and polarity by myosins IIA and IIB in migrating cells. J Cell Biol, 176, 573-80.

Wang B, Yan Y, Zhou J, et al (2013a). A novel all-trans retinoid acid derivatives inhibits the migration of breast cancer cell lines MDA-MB-231 via myosin light chain kinase involving p38-MAPK pathway. Biomed Pharmacother, 67, 357-62.

Wang H, Gui SY, Chen FH, et al (2013b). New Insights into 
4-Amino-2-tri-fluoromethyl-phenyl Ester Inhibition of Cell Growth and Migration in the A549 Lung Adenocarcinoma Cell Line. Asian Pac J Cancer Prev, 14, 7265-70.

Wang J, Guo W, Chen W, et al (2013c). Melatonin potentiates the antiproliferative and pro-apoptotic effects of ursolic acid in colon cancer cells by modulating multiple signaling pathways. J Pineal Res, 54, 406-16.

Wang J, Hao H, Yao L, et al (2012d). Melatonin suppresses migration and invasion via inhibition of oxidative stress pathway in glioma cells. J Pineal Res, 53, 180-7.

Wenzel U, Nickel A, and Daniel H (2005). Melatonin potentiates flavoneinduced apoptosis in human colon cancer cells by increasing the level of glycolytic end products. Int J Cancer, 116, 236-42.

Wilson AK, Gorgas G, Claypool WD, and de Lanerolle P(1991). An increase or a decrease in myosin II phosphorylation inhibits macrophage motility. J Cell Biol, 114, 277-83.

Winczyk K, Fuss-Chmielewska J, Lawnicka H, et al (2009). Luzindole but not 4-phenyl-2- propionamidotetralin (4P-PDOT) diminishes the inhibitory effect of melatonin on murine Colon 38 cancer growth in vitro. Neuro Endocrinol Lett, 30, 657-62.

Yan X, Rui X, and Zhang K (2015). Baicalein inhibits the invasion of gastric cancer cells by suppressing the activity of the p38 signaling pathway. Oncol Rep, 33, 737-43.

Yi C, Zhang Y, Yu Z, et al (2014). Melatonin enhances the antitumor effect of fisetin by inhibiting COX-2/iNOS and NFkB/p300 signaling pathways. PLoS One, 9, 99943.

Yun M, Kim EO, Lee D, et al (2014). Melatonin sensitizes H1975 non-small-cell lung cancer cells harboring a T790M-targeted epidermal growth factor receptor mutation to the tyrosine kinase inhibitor gefitinib. Cell Physiol Biochem, 34, 865-72.

Zha L, Fan L, Sun G, et al (2012). Melatonin sensitizes human hepatoma cells to endoplasmic reticulum stress-induced apoptosis. J Pineal Res, 52, 322-31.

Zhou Q, Gui S, Zhou Q, et al (2014a). Melatonin Inhibits the Migration of Human Lung Adenocarcinoma A549 Cell Lines Involving JNK/MAPK Pathway. PLoS One, 9, 101132.

Zhou X, Liu Y, You J, et al (2008b). Myosin light-chain kinase contributes to the proliferation and migration of breast cancer cells through cross-talk with activated ERK1/2. Cancer Lett, 270, 312-27.

Zhu P, Chen JM, Guo HM, et al (2012). Matrine inhibits disturbed flow-enhanced migration via downregulation of ERK1/2-MLCK signaling vascular smooth muscle cells. Ann Vasc Surg, 26, 268-75. 